\title{
Optical spectroscopy and microscopy of radiation- induced light-emitting point defects in lithium fluoride crystals and films
}

\author{
R.M. Montereali ${ }^{1}$, F. Bonfigli ${ }^{1}$, F. Menchini ${ }^{2}$, and M.A. Vincenti ${ }^{1}$ \\ ${ }^{I}$ ENEA C.R. Frascati, Photonics Micro- and Nano-structures Lab., UTAPRAD-MNF \\ Via E. Fermi 45, 00044 Frascati (Rome), Italy \\ E-mail: rosa.montereali@enea.it \\ ${ }^{2}$ ENEA C.R. Casaccia, Optical Coatings Laboratory, UTTMAT-OTT \\ Via Anguillarese 301, 00123 S. Maria di Galeria (Rome), Italy
}

Received April 23, 2012

\begin{abstract}
Broad-band light-emitting radiation-induced $F_{2}$ and $F_{3}{ }^{+}$electronic point defects, stable and laser-active at room temperature in lithium fluoride crystals and films, find applications in dosimeters, tuneable color-center lasers, broad-band miniaturized light sources and in novel radiation imaging detectors. A brief review of their photoemission properties is presented, and their peculiarities at liquid nitrogen temperature are discussed. A few experimental results about optical spectroscopy and fluorescence microscopy of these radiation-induced point defects in LiF crystals and thin films are presented to obtain information about the coloration curves, the point defects formation efficiency, the effects of the photo-bleaching processes, and so on. The control of local formation, stabilization and transformation of radiation-induced light-emitting defect centers is crucial for the development of optical active micro-components and nanostructures. Some of the advantages of low temperature measurements for novel confocal laser scanning fluorescence microscopy techniques, widely used for the spatial mapping of these point defects thorough the optical reading of their visible photoluminescence, are highlighted.
\end{abstract}

PACS: $61.72 . j n$ Color centers.

Keywords: photoluminescence, color centers, optical microscopy, thin films, alkali halides.

\section{Introduction}

Light-emitting radiation-induced point defects in lithium fluoride, LiF, [1] find applications in dosimeters [2-4], tunable color-center lasers [5-7], broad-band miniaturized light sources [7-9] and in novel radiation imaging detectors based on the optical reading of photoluminescence (PL) from $F_{2}$ and $F_{3}^{+}$aggregate color centers [10-13]. These different types of laser-active centers, consisting of two electrons bound to two and three adjacent anion vacancies, respectively, possess almost overlapping absorption bands peaking at $\sim 450 \mathrm{~nm}$, generally called $M$ band [1], and Stokes-shifted broad photoemission bands centered at $678 \mathrm{~nm}$ and $535 \mathrm{~nm}[1,14]$, respectively. Their high emission efficiencies [6] and lasing characteristics [5] make further investigations about the optical properties of these electronic defects relevant.

$\mathrm{LiF}$ possesses the largest band gap, greater than $14 \mathrm{eV}$, of any solid, with the possible exception of exotic materials such as solid rare gases; therefore its high optical transparency is particularly suitable for the investigation of lat- tice defect features by optical spectroscopy and advanced fluorescence microscopy techniques $[15,16]$ to derive information about radiation effects on this interesting material in different forms.

High optical quality pure and doped LiF crystals as well as transparent polycrystalline LiF thin films [8] can be grown by several methods. They constitute the host matrix for the broad-band visible-emitting $F_{2}$ and $F_{3}^{+}$radiationinduced defects, which can be used as a probe of the host matrix characteristics [17]. On the other hand, LiF-based photonic micro-components $[7,9,18]$ and nanostructures $[8,16,19]$ with peculiar optical active properties can be produced by the control of the local formation, stabilization and photo-thermal transformation of radiation-induced light-emitting defect centers.

The main spectral characteristics of $F_{2}$ and $F_{3}^{+}$point defects in $\operatorname{LiF}[1,14]$, i.e. broad optical absorption and stokes-shifted broad photoluminescence bands, are typical of color centers in alkali halides and quite well known from the literature [20]. With respect to other alkali hal- 
ides, a peculiarity of radiation effects in $\mathrm{LiF}$ is the stability of these point defects also at room temperature (RT) and the stable formation of high densities of $F_{3}^{+}$centers, together with the $F$ and $F_{2}$ defects [21]. This behavior is still more significant for low-temperature irradiation, where the $F_{3}^{+}$center formation is preferred $[14,22]$. Moreover, the presence of impurities in the LiF matrix strongly influences their formation and stabilization processes.

The $F_{2}$ and $F_{3}^{+}$overlapping optical absorption bands, together with other specific behaviors, such us the presence of a metastable triplet state in the optical cycle [23], sometimes make it difficult, or tricky, to perform a direct comparison of PL spectra of different LiF samples, which could be helpful in order to get information about the effects of irradiation conditions [14,21,22], the host material characteristics [17] and composition, and the emission properties of novel LiF based photonic micro- and nanostructures $[8,9,12,18]$. In particular, the present interest in the miniaturization of photonic components has stimulated the use of thin films for broad-band light-emitting devices. It is crucial to establish the influence that the LiF film growth parameters have on the stabilization of electronic defects in polycrystalline thin layers in comparison with single crystals [17]. These complex phenomena are currently under investigation in many dielectric host matrix and nanomaterials prepared by different methods.

Recently the broad-band light-emitting properties of $F_{2}$ and $F_{3}^{+}$CCs in LiF crystals [1] and films [8] found promising application as radiation imaging detectors characterized by very high spatial resolution $(\sim 250 \mathrm{~nm})$ on a wide field of view $\left(>1 \mathrm{~cm}^{2}\right)$. The intrinsic spatial resolution is in principle limited only by the size of point defects, which is at atomic scale. This means that the resolution could be below a few nanometers [24], since the lattice constant of LiF is only $0.2013 \mathrm{~nm}$.

In practice, the spatial resolution is essentially limited by the optical microscope and the technique utilized for PL detection in the optical reading process. The LiF crystals and films sensitivity to EUV [11], soft [10] and hard $\mathrm{x}$ rays [12], as well as to thermal neutrons [13], enables advanced radiation imaging applications in table-top systems [10-12] as well as in large scale facilities $[13,19]$. The direct writing of micro- and nanostructures by focused ionizing radiation $[16,19]$ and laser $[9,18]$ beams and the transfer of luminescent micro-patterns by lithographic masks [11] is under way for research and developments in nano-bio-photonics.

Laser induced optical fluorescence scanning microscopy in far-field confocal configurations $[12,15]$ as well as in near-field approach [16] was successfully applied to twodimensional mapping of $F_{2}$ and $F_{3}^{+}$aggregate defects in $\mathrm{LiF}$ matrix at sub-micrometric and nanometric scale, respectively. Confocal fluorescence microscopy techniques combined with micro-luminescence measurements were used for three-dimensional reconstruction and identification of the spatial distribution of several kinds of color centers [15].
Some of these aspects are presented, discussed and reviewed in this paper, on the basis of different examples, in order to highlight some of the peculiarities of optical spectroscopy at liquid nitrogen temperature (LNT) and their interesting perspectives for the spectral investigation of optically active color centers in LiF crystals and films by advanced optical fluorescence microscopy techniques proposed and tested for high-resolution imaging of point defects in insulating materials $[25,26]$.

\section{Optical absorption spectra of radiation induced point defects in LiF crystals}

Unlike other alkali halides, CCs can be created in LiF only by ionizing radiations, like energetic photons including gamma [14] and hard $\mathrm{x}$ rays [1,12], as well as high-energy particles, including charged electrons [22] and ions, and neutrons [13], but also by low-penetrating radiations, like soft $\mathrm{x}$ rays [10,19], low-energy electrons [15-17] and light ion beams. Recently their stable formation was obtained in $\mathrm{LiF}$ crystals also by intense ultra-short laser pulses [9].

The radiation-induced point defects mainly consist of primary $F$ centers and aggregate $F_{2}$ and $F_{3}^{+}$point defects, whose absolute and relative concentrations are dependent on the specific radiation type and the selected irradiation conditions. Generally optical absorption measurements are suitable for color centers monitoring in transparent LiF crystals. As an example, Fig. 1 shows the optical absorption spectra of a $2 \mathrm{~mm}$ thick LiF crystal, gamma-irradiated by a ${ }^{60} \mathrm{Co}$ source, dose $4.2 \cdot 10^{4} \mathrm{~Gy}$, measured at RT and LNT. The crystal is mounted under vacuum in the sample holder of an optical cryostat operating at RT and at LNT, and the absorption spectrum of the two opposite silica windows is also reported for comparison.

The most important spectral features are the $M$ band and the $F$ one, which appears truncated because of the typical

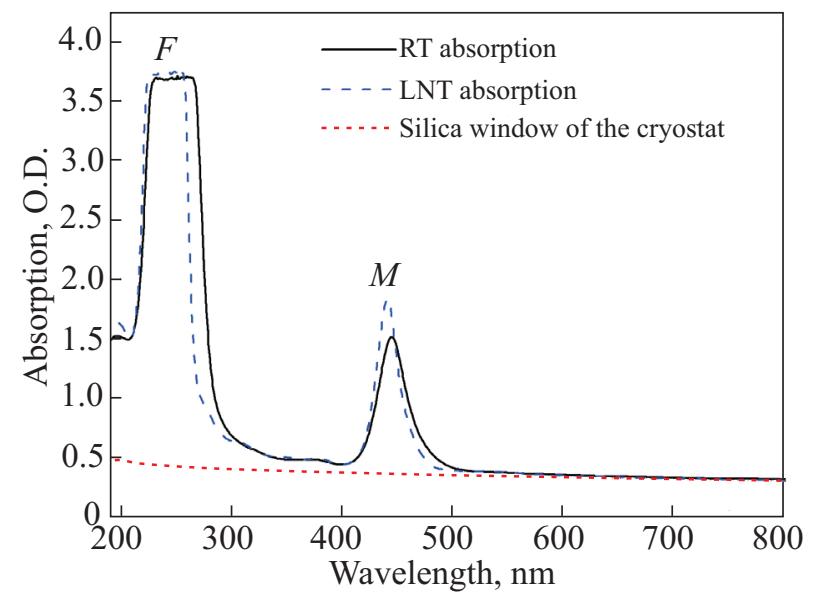

Fig. 1. Optical absorption spectra of a $2 \mathrm{~mm}$ thick gammairradiated LiF crystal, dose $4.2 \cdot 10^{4} \mathrm{~Gy}$, measured in the holder of a cryostat under vacuum at RT (solid line) and at LNT (dashed line). The absorption spectrum of the silica window of the optical cryostat (dotted line) is also shown. 
transmission detection limit of standard spectrophotometers. According to the literature, at RT, the primary $F$ absorption peak is at around $243 \mathrm{~nm}$ [20], while the position of the $M$ band, peaked around $450 \mathrm{~nm}$, is dependent on the ratio between the $F_{2}$ and $F_{3}^{+}$volume concentrations.

Due to the strong electron-phonon coupling to the lattice, the absorption bands are homogeneously broadened [20]. Lowering the temperature, the peak positions shift towards higher energies and their full widths at half maximum (FWHMs), are reduced; the intensities increase in order to keep constant the total area of each absorption band associated to one kind of point defect, according to the physical meaning stated by the Smakula expression [20]. The spectral features of the $F$ band are well known from the literature. Due to the full overlapping of the $F_{2}$ and $F_{3}^{+}$absorption bands, the situation is more complex for these two kinds of point defects. Different values of peak positions and FWHMs are found in the literature. Suitable values were found in [14], by a careful comparison of optical absorption spectra of several gamma irradiated polished LiF crystals, whose irradiation parameters (dose and temperature) and subsequent thermo-optical treatments were properly chosen in order to get the preferential formation of mainly one kind of these defects. The polished LiF slices of equal thickness were obtained from a nominally pure LiF single crystal and the optical absorption and PL measurements were performed at RT and LNT in the same experimental conditions, assuming Gaussian line shape for the best fit procedure. The obtained results are summarized in Table 1 and will be used as reference values in the following. Figure 2 shows the results of the best-fit procedure by these Gaussian curves of the $M$ absorption band, measured at LNT, of the same gammacolored LiF crystal in Fig. 1. The O.D. spectrum of a blank LiF crystal, a LiF crystal with the same geometric dimensions and surface polishing quality, but non-irradiated, was subtracted to the measured spectrum, in order to highlight the effects of the coloration and reduce spurious contributions. The peak position of the $F_{3}^{+}$ absorption band at $2.77 \mathrm{eV}(448 \mathrm{~nm})$ is practically the same both at RT and at LNT; in the case of $F_{2}$ color

Table 1. Values of the peak position $\left(E_{a}, E_{e}\right)$ and the full width at the half maximum $\left(\mathrm{FWHM}_{a}, \mathrm{FWHM}_{e}\right)$ of the optical absorption and emission bands of $F_{2}$ and $F_{3}^{+}$CCs in LiF at RT and at LNT [from Ref. 14].

\begin{tabular}{|c|c|c|c|c|c|}
\hline \multirow[b]{2}{*}{ Center } & \multirow[b]{2}{*}{ Temperature } & \multicolumn{4}{|c|}{ Gaussian band parameters } \\
\hline & & $\begin{array}{l}E_{a} \\
\mathrm{eV}\end{array}$ & $\begin{array}{c}\mathrm{FWHM}_{a} \\
\mathrm{eV}\end{array}$ & $\begin{array}{l}E_{e} \\
\mathrm{eV}\end{array}$ & $\begin{array}{c}\mathrm{FWHM}_{e}, \\
\mathrm{eV}\end{array}$ \\
\hline \multirow{2}{*}{$F_{2}$} & RT & 2.79 & 0.16 & 1.83 & 0.36 \\
\hline & LNT & 2.82 & 0.13 & 1.84 & 0.29 \\
\hline \multirow{2}{*}{$F_{3}^{+}$} & RT & 2.77 & 0.29 & 2.29 & 0.31 \\
\hline & LNT & 2.77 & 0.23 & 2.33 & 0.22 \\
\hline
\end{tabular}

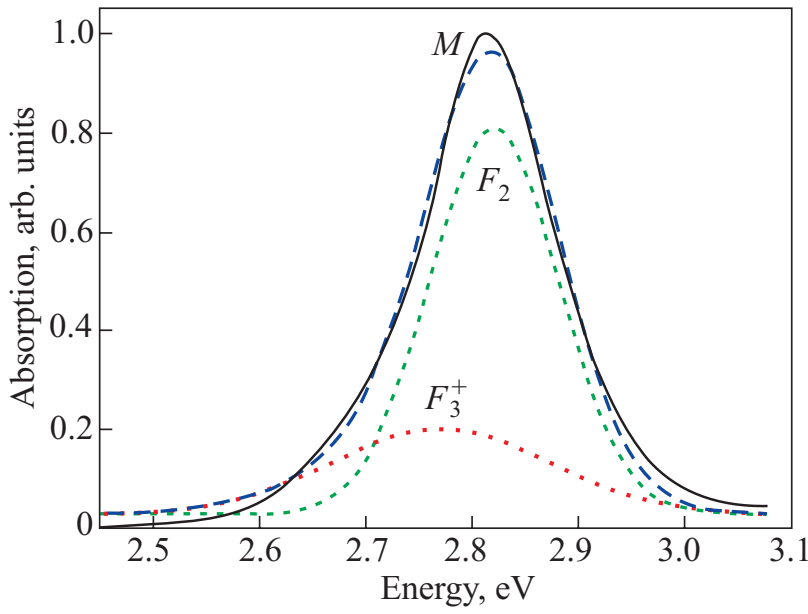

Fig. 2. LNT absorption spectrum (solid line) and best-fit (dashed line) of the $M$ band of the same gamma-colored LiF crystal shown in Fig. 1. The partial Gaussian contributions of $F_{2}$ and $F_{3}^{+}$(dotted lines) CCs obtained by the best-fit are shown.

centers, the peak position shifts from $2.79 \mathrm{eV}$ (444 nm) towards higher energies, $2.82 \mathrm{eV}$, i.e. lower wavelengths $(440 \mathrm{~nm})$, at LNT.

According to the optical absorption best-fit results reported in Fig. 2, on the basis of the oscillator strength values [1], it is possible to derive the volume concentrations of $F_{2}$ and $F_{3}^{+}$CCs in the gamma-irradiated LiF crystals [14], whose coloration is uniform along the thickness.

The volume densities values allow to study the $F, F_{2}$ and $F_{3}^{+}$coloration curves and the relevant effects of several irradiation parameters, in order to tailor the photoemission properties of the irradiated material. It is well known that lowering the irradiation temperature reduces the formation efficiency of the primary $F$ centers, and then of the $F_{2}$ ones. On the contrary, at low temperature, the stable formation of $F_{3}^{+}$defects is favoured with respect to $F_{2}$ ones, and the ratio between their concentrations increases. This behavior was obtained also in the case of high energy $(3 \mathrm{MeV})$ electron irradiation of LiF crystals [22], as will be shown below.

\section{Photoluminescence spectra of light-emitting point defects in LiF crystals}

Laser induced PL spectra under optical excitation in the $M$ absorption band are also very effective to probe the $F_{2}$ and $F_{3}^{+}$stable formation in irradiated LiF crystals, due to the simultaneous excitation of both kinds of optically active CCs. As PL is not an absolute measure, experimental care should be taken in order to compare different colored LiF samples. In the case of broad-band light-emitting $F_{2}$ and $F_{3}^{+} \mathrm{CCs}$ in $\mathrm{LiF}$, the selected measurement conditions strongly affect the result of the measurements. Some of these effects are briefly analyzed in the following. As an example, Fig. 3 shows the PL spectra at RT and LNT of a 


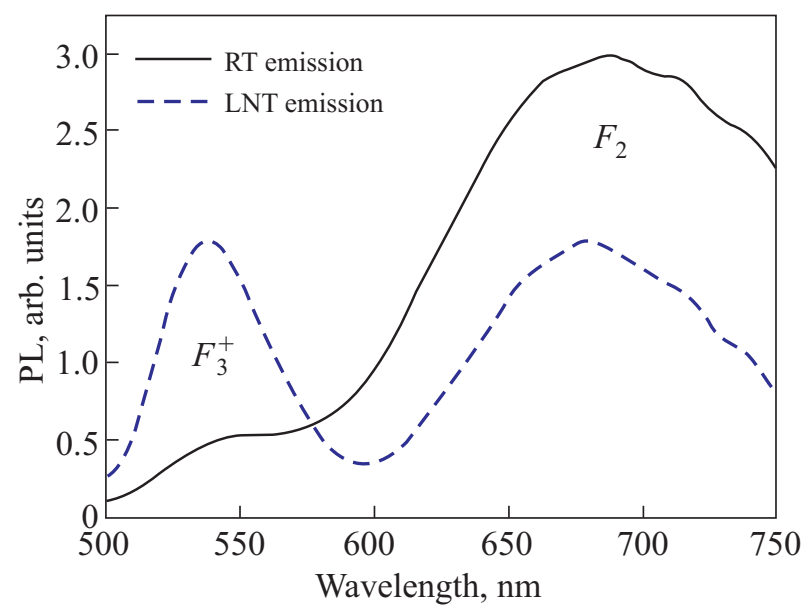

Fig. 3. PL spectra of a gamma-irradiated LiF crystal, dose 8.4 $10^{2} \mathrm{~Gy}$, at RT (solid line) and LNT (dashed line) under laser excitation at the wavelength of $457.9 \mathrm{~nm}$.

gamma-irradiated LiF crystal, dose $8.4 \cdot 10^{2}$ Gy, excited by the Argon laser line $457.9 \mathrm{~nm}(2.71 \mathrm{eV})$. The PL peak intensity is lower at LNT than at RT for $F_{2} \mathrm{CCs}$, while it increases for $F_{3}^{+}$ones. This behavior is a direct consequence of the spectral characteristics of $F_{2}$ and $F_{3}^{+}$CCs in LiF. According to the values reported in Table 1, the shift of the peak position for the $F_{2}$ absorption bands towards higher energies is accompanied by a small reduction in its FWHM with respect to the $F_{3}^{+}$one; however, as the FWHM of $F_{2}$ absorption band is much lower than that of the $F_{3}^{+}$one, the ratio of the $F_{2}$ to $F_{3}^{+}$emission intensities is reversed changing the measurement temperature for the same colored LiF crystal at a fixed excitation wavelength. So, at a specific excitation wavelength, the intensity ratio of their emission intensities is obviously dependent on the temperature.

As a direct consequence of this behavior, also the choice of the excitation wavelength in the broad $M$ absorption band strongly influences the $F_{2}$ to $F_{3}^{+}$emission intensities ratio in the PL spectra of LiF crystals and thin films [8]. Obviously, this effect is still more marked for LNT measurements.

\section{Radiation induced $F_{2}$ and $F_{3}^{+}$color centers in LiF crystals and thin films}

As already stated, only the optical absorption measurements allow a direct quantitative evaluation of CCs concentrations in entirely colored LiF crystals. In order to compare the effects of the irradiation temperature on the selective formation of $F_{2}$ and $F_{3}^{+}$lasing CCs, two $\mathrm{LiF}$ crystals of different thickness were irradiated by a $3 \mathrm{MeV}$ electron beam at two different temperatures, RT and $213 \mathrm{~K}$ [22]. The sample irradiated at RT with a dose of $4.5 \cdot 10^{4} \mathrm{~Gy}$, 3.5 times lower than the one irradiated at $213 \mathrm{~K}$, has a comparable absorption coefficient, about $18 \mathrm{~cm}^{-1}$, on the peak of the $\mathrm{M}$ absorption band, as shown in Figs. 4, $a$ and 5, $a$ for LNT absorption spectra. In Figs. $4, b$ and 5,b their normalized PL spectra, laser excited at $457.9 \mathrm{~nm}$ at LNT, are reported together with their decomposition in two Gaussian emission bands arising from $F_{2}$ and $F_{3}^{+}$centers, according to Table 1.

The PL spectra were obtained under laser excitation at low power density to reduce the effect of trapping of $\mathrm{F}_{3}^{+}$ excited population by a metastable triplet state [23], which decreases the green emission intensity under intense optical pumping; this effect induces a sub-linear increase of the $F_{3}^{+}$color centers PL intensity as a function of the pumping power and it is much stronger at RT rather than at LNT.
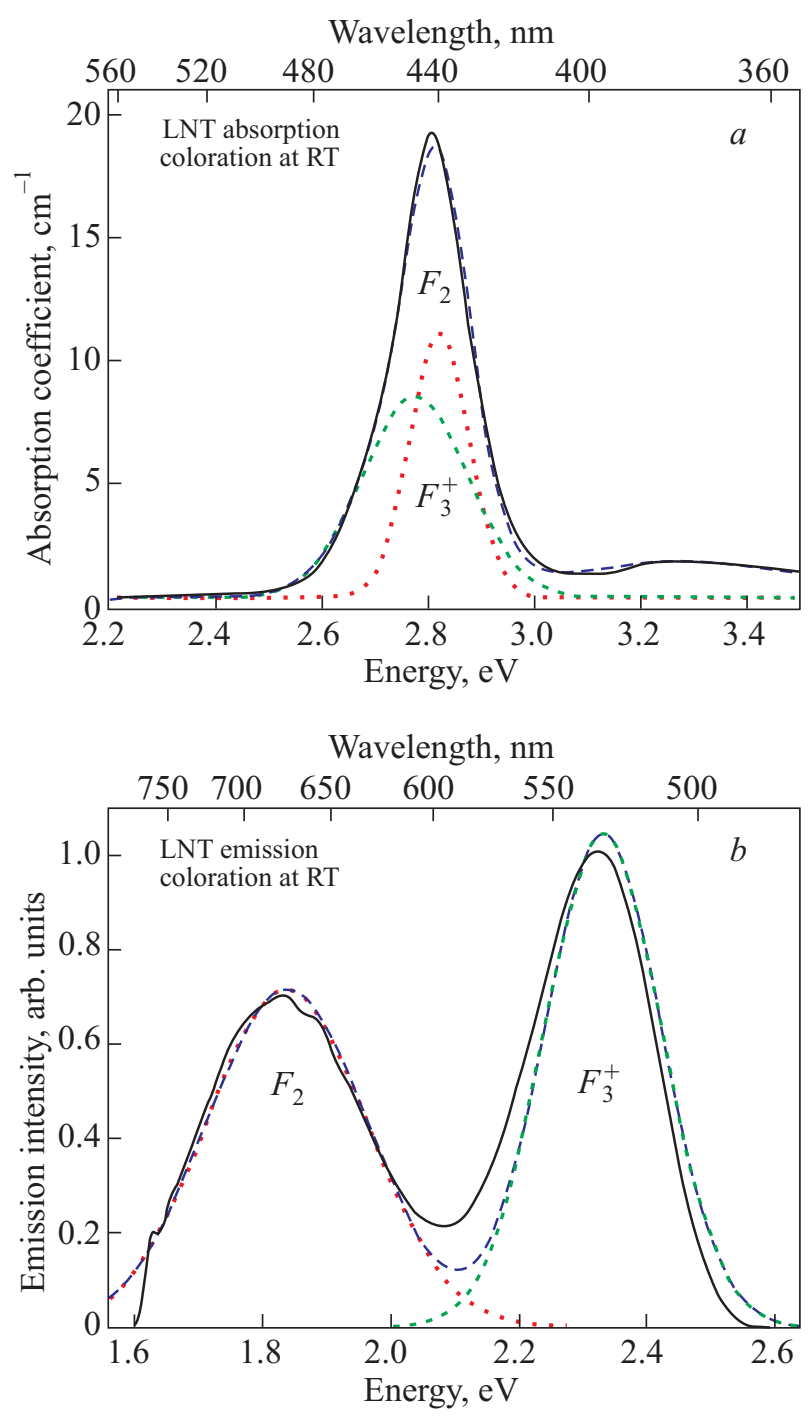

Fig. 4. LNT absorption coefficient (solid line) and best-fit (dashed line) of the $M$ absorption band of a LiF crystal irradiated by a $3 \mathrm{MeV}$ electron beam at RT with a dose $4.5 \cdot 10^{4} \mathrm{~Gy}(a)$ and LNT PL spectrum (solid line) under laser excitation at the wavelength of $457.9 \mathrm{~nm}$ and best fit (dashed line) (b). The experimental data (solid lines) have been fitted as the sum (dashed lines) of Gaussian bands (dotted lines). 

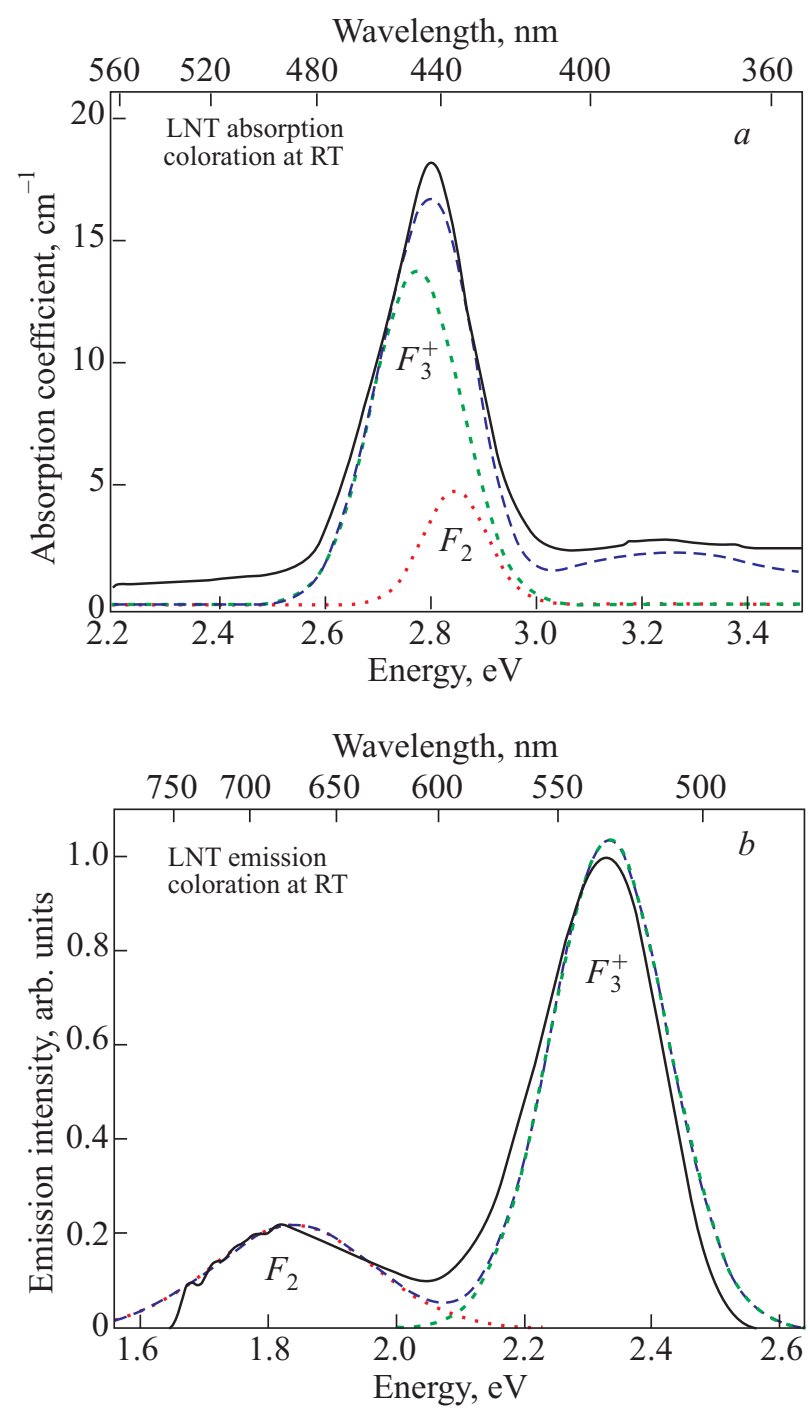

Fig. 5. LNT absorption coefficient (solid line) and best-fit (dashed line) of the $M$ absorption band of a LiF crystal irradiated by a $3 \mathrm{MeV}$ electron beam at $213 \mathrm{~K}$ with a dose $1.6 \cdot 10^{5} \mathrm{~Gy}(a)$ and LNT PL spectrum (solid line) under laser excitation at the wavelength of $457.9 \mathrm{~nm}$ and best fit (dashed line) (b). The experimental data (solid lines) have been fitted as the sum (dashed lines) of Gaussian bands (dotted lines).

The PL fitting results in Figs. $4, b$ and $5, b$ were not as good as in the case of optical absorption spectra, probably due to the fact that a third emission band, peaked around $610 \mathrm{~nm}(2.03 \mathrm{eV})$, should be taken into account. This emission band, tentatively attributed to perturbed $F_{2}$ centers, $F_{2}^{p}$, is due to the unintentional presence of impurities, like oxygen and magnesium, inside the crystal matrix. It proved to be quite important because it has been successfully used to obtain a yellow-orange laser emission at RT [27]. On the other hand, according to the combined excitation-emission spectroscopic investigation performed at LNT [28], its negligible absorption contribution, peaked at around $495 \mathrm{~nm}(2.51 \mathrm{eV})$, practically does not affect the best fit of the $M$ absorption band in Figs. 4, $a$ and 4, $b$.
From the comparison of the LNT normalised PL spectra in Figs. $4, b$ and 5, $b$ we can notice a relative increase of the $F_{3}^{+}$band with respect to the $F_{2}$ one for high energy electrons irradiation at low temperature. A systematic study [22] of their optical absorption spectra demonstrated that the formation efficiency of these aggregate defects at $213 \mathrm{~K}$ is reduced by about one order of magnitude with respect to irradiation at $\mathrm{RT}$, but the volume density of stable $F_{3}^{+}$color center is more than the double of the $F_{2}$ one. This behavior is at the basis of preparation methods of suitable lasing media for LiF-based solid-state green lasers, in order to reduce the optical losses due to pump absorption by $F_{2}$ defects. The low temperature irradiation is characterized by a substantial reduction of more complex aggregate defects, which is also a relevant feature to reduce the green $F_{3}^{+}$emission re-absorption phenomena by parasitic centers $[5,8]$. The overall decrease of defect formation efficiency is clearly associated with a reduced interstitial mobility at low temperatures, which halts the stabilization of the $F$ centers by slowing down the aggregation processes.

In the case of $\mathrm{LiF}$ thin films on transparent substrates, the strong overlapping of the $F_{2}$ and $F_{3}^{+}$absorption bands is further complicated by the presence of interference fringes in their optical absorption spectra [8] related to the refractive index difference between the LiF film and the substrate. For this reason, the easiest and direct way of analysing their formation and characteristics is by the PL spectra measurements, which can be applied also to the case of LiF films grown on not-transparent substrates [8].

Again, this approach can be used to investigate the influence of the irradiation conditions on the concentration ratio of $F_{2}$ to $F_{3}^{+}$centers. As an example, $\mathrm{PL}$ of $\mathrm{LiF}$ films thermally evaporated on glass substrates in the same growth conditions and irradiated by $3 \mathrm{keV}$ electrons at RT, $-60{ }^{\circ} \mathrm{C}$ and LNT are reported in Fig. 6 .

As the $3 \mathrm{keV}$ electron penetration depth in $\mathrm{LiF}$ is estimated to be approximately $150 \mathrm{~nm}$ [8], films thicker than $150 \mathrm{~nm}$ were irradiated, in order to allow a direct comparison of their PL spectra, performed in the same experimental conditions. Although the colored thickness was quite low, the high concentrations of $F_{2}$ and $F_{3}^{+}$defects obtained in the thin colored surface layer [29] and the sensitivity of the laser excited PL detection lock-in technique allow to obtain a high signal to noise ratio. At LNT the PL signal is higher and the $F_{2}$ and $F_{3}^{+}$spectral contributions are well separated, so a direct comparison is possible. Again, lowering the irradiation temperature, the $F_{3}^{+}$to $F_{2}$ ratio increases, and the defects formation efficiency is reduced. Moreover, from a careful comparison between the PL spectra of the film colored at $-60{ }^{\circ} \mathrm{C}$ and a $\mathrm{LiF}$ crystal irradiated in the same conditions, it emerges that the film PL signal is from 3 to 5 times more intense, depending on the kind of aggregate defects [29]. The higher coloration efficiency of thin films is connected to their high surface to volume ratio related to granular structure. The grain 


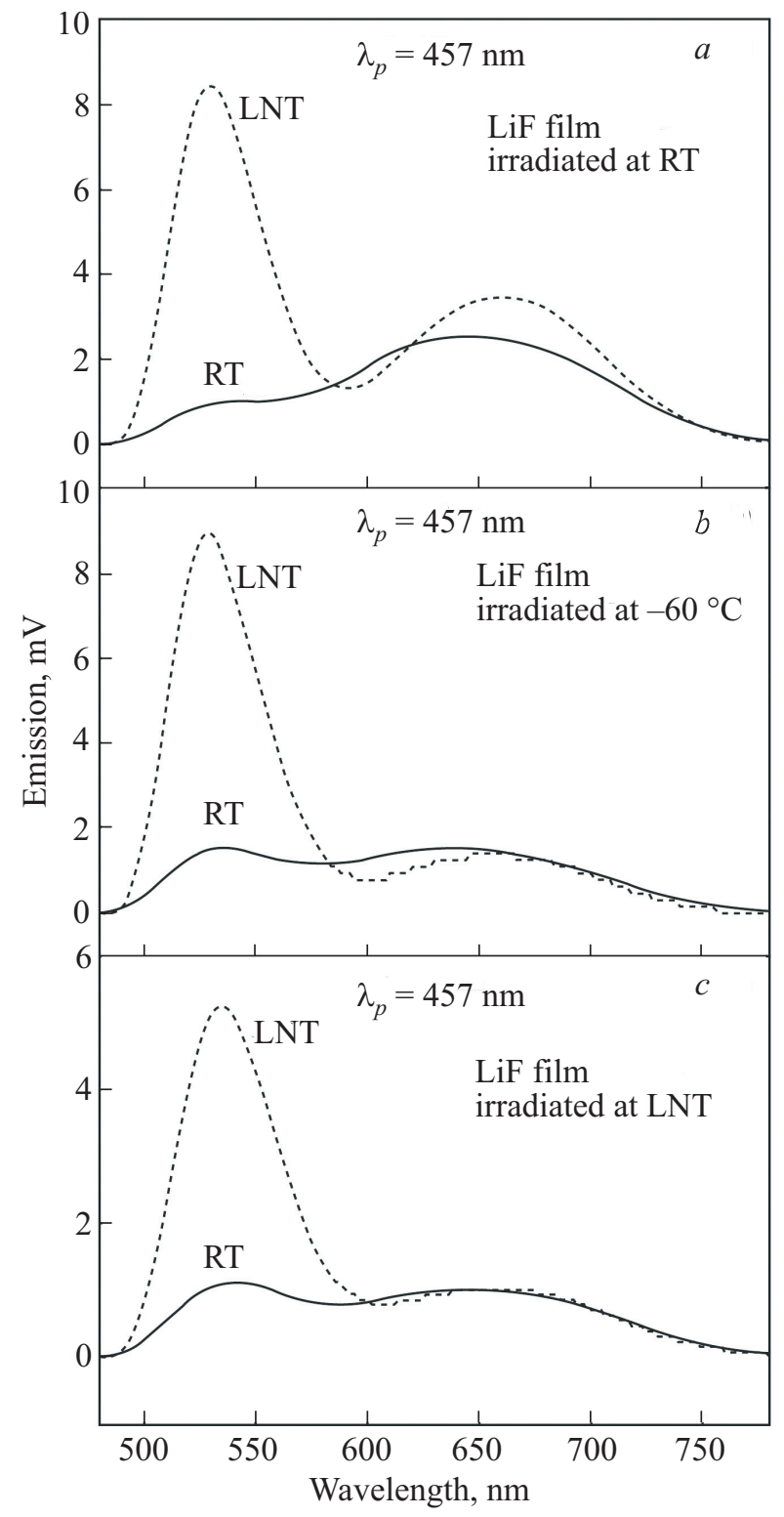

Fig. 6. PL spectra of LiF films thermally evaporated on glass substrates and irradiated by a $3 \mathrm{keV}$ electron beam at RT $(a),-60{ }^{\circ} \mathrm{C}$ (b), and LNT (c), measured at RT (solid lines) and LNT (dashed lines) under laser excitation at the wavelength of $457.9 \mathrm{~nm}$.

boundaries in the polycrystalline $\mathrm{LiF}$ matrix act as a source of vacancies in the initial stage of coloration, and favor the formation of surface traps, which are essential for the coloring process, by stabilising the interstitial ions. The compactness of the film should also play a relevant role because it establishes the density of the grain boundaries, which act as a source of vacancies during the CCs formation and stabilization processes [17]. It is well known that vacancies, or lattice point defects, are created much more easily in nanomaterials with respect to their bulk counterparts. It is important to control how vacancies are formed as the size of grains becomes smaller and it seems that vacancies are easier to form in particles of reduced size compared to the bulk. These behavior is rather intuitive, as vacancies are thought of as being related to the internal surface in a material, and it is therefore natural to expect that decreasing the size of a material will increase the concentration of vacancies in it, thanks to an increasing surface area to volume ratio.

\section{Optical fluorescence microscopy of light-emitting color centers in LiF based micro-components and nanostructures}

The present trends towards devices miniaturization and the nanotechnologies progress make attractive the investigation of radiation induced $F_{2}$ and $F_{3}^{+}$color centers in LiF based light-emitting nanostructures and micro-components, as well as their local modifications by selective laser tools. In these cases, it is necessary to perform a local mapping of point defects spatial distribution and to investigate their properties by optical microscopy and micro-luminescence measurements. The $F_{2}$ and $F_{3}^{+}$CCs broad absorption and emission bands in the visible spectral range and their high PL efficiency allow the collection of enough signal even from small colored volumes. In principle, by increasing the sensitivity of the PL measurement system, even a single photon is detectable. Moreover novel advanced lens-based fluorescence microscopy techniques, with a resolution no longer limited by diffraction, demonstrated imaging of extrinsic color centers in insulating materials with nanoscale resolution and Angstrom precision [26].

In order to analyze the effects of the sample preparation procedures on the light-emission characteristics of the color centers LiF-based micro-structures, reliable information can be obtained by exploiting the simultaneous optical excitation with a single blue wavelength in the $M$ absorption band and the separate collection of spectrally integrated red $F_{2}$ and green $F_{3}^{+}$CCs photoluminescence. As an example, Fig. 7 shows the confocal fluorescence image of regular lightemitting structures at nanoscale, obtained on a LiF crystal by direct writing with the focused soft $\mathrm{x}$ ray beam provided at the ELETTRA synchrotron radiation facility [19]. The beam energy is $650 \mathrm{eV}$, which corresponds to an attenuation length in $\mathrm{LiF}$ of about $4 \mu \mathrm{m}$; the colored area, $56 \times 62 \mu \mathrm{m}$ wide, forms a regular pattern, which consists of photoluminescent lines regularly spaced by $1 \mu \mathrm{m}$, whose FWHM, about $340 \mathrm{~nm}$, was obtained from the grey level PL signal intensity profile of image in Fig. 7. The used confocal laser scanning microscope is equipped with a laser operating at $457.9 \mathrm{~nm}$ and the integrated emitted fluorescence was collected with a $60 \mathrm{x}$, N.A. $=1.4$ objective immersed in oil and detected by means of a two channel photomultiplier system, which allows simultaneously acquiring the red and green signals coming from each channel, selected by suitable optical filters that also cut off the pump blue light. The sectioning capability of the CLSM is crucial to reach this lateral spatial resolution value, which is well below the wavelength of the collected emitted radiation. 


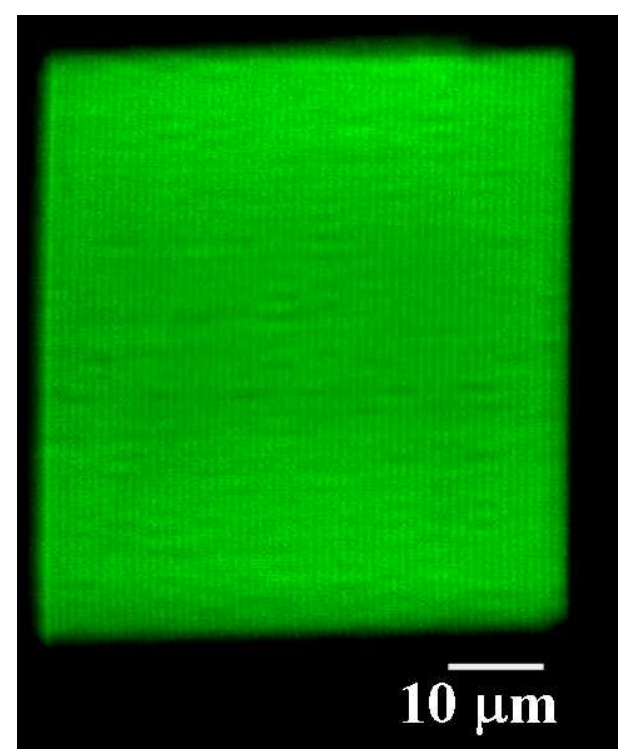

Fig. 7. Confocal fluorescence image of permanent, regular, color center lines directly written on a LiF crystal by a focused soft $\mathrm{x}$ ray beam, measured under laser excitation at the wavelength of $457.9 \mathrm{~nm}$ (see text for details).

Another example is the recording of permanent Bragg gratings on entirely gamma colored LiF crystals, obtained by using the interference patterns of a continuous-wave laser [18]. Under low-power illumination with monochromatic light at $244 \mathrm{~nm}$, close to the peak wavelength of the $F$ absorption band in LiF, the selective bleaching of primary $F$ centers and the preferential bleaching of aggregate $F_{2}$ laser active defects were identified as the main mechanisms leading to permanent recording of regular light-emitting micro-patterns of different spatial periods from 400 to 1000 $\mathrm{nm}$. The laser-writing method was successfully extended to a LiF crystal irradiated by extreme ultra-violet and soft $\mathrm{x}$ rays in order to obtain an ultra-thin densely colored surface layer, which thickness was estimated to be lower than $50 \mathrm{~nm}$ [30]. The ultraviolet (UV) laser-induced selective optical bleaching of primary and aggregate defects is responsible for the periodic spatial modulation of optical absorption and PL properties. Optical absorption and fluorescence spectra confirm the photo-bleaching of $F$-aggregate color centers as a result of the laser recording process. The RT PL spectra, measured in the same experimental conditions, of two gratings recorded with the same power for different illumination times (10 and 20 min, respectively) are shown in Fig. 8, together with the visible PL spectrum obtained on the gamma colored $\mathrm{LiF}$ area before UV illumination.

They consist of two broad emission bands, which were successfully best-fitted with the sum of two Gaussian curves, whose peak wavelengths and bandwidths are consistent with the literature [14]. Big differences before and after UV illumination are noticeable: both the $F_{3}^{+}$and $F_{2}$ emission intensities are strongly reduced due to the UV induced bleaching. However, the reduction is more dramatic for the

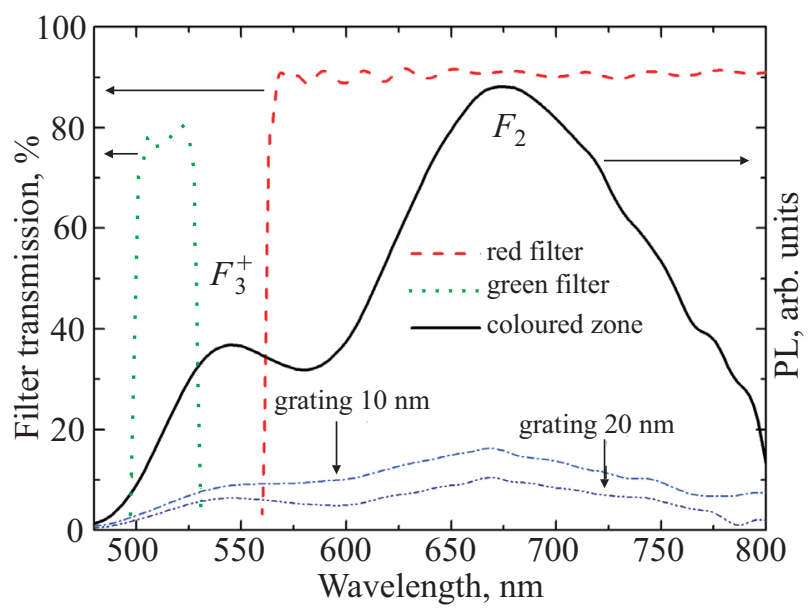

Fig. 8. RT photoluminescence spectra of the surface coloured zone of a LiF crystal (solid line) and of the two gratings (dashdotted lines) recorded for two illumination times, measured under laser excitation at the wavelength of $457.9 \mathrm{~nm}$. The transmission spectra of the optical filters mounted in the fluorescence collection system of the confocal laser scanning microscope used to measure the pitch of the recorded gratings are also shown (see text for details).

$F_{2}$ band; this fact suggests that the UV excitation at $244 \mathrm{~nm}$, in the peak of $F$ band absorption, is quite a selective mechanism to bleach $F_{2}$ centers more efficiently. In Fig. 8, the transmission spectra of the optical filter mounted in the collection system of the confocal laser scanning microscope are also reported. They were chosen in order to isolate and measure the $F_{2}$ and $F_{3}^{+}$color centers PL spectral contributions, avoiding spurious signals from their superposition. This approach is suitable in order to derive information about the nature of the radiation-induced defects and their photo-thermal transformation under laser irradiation, specially on luminescent objects characterized by subwavelength dimensions, like in the case of the confocal fluorescence image in Fig. 7. It should be noticed that, also in this case of soft $\mathrm{x}$-ray irradiation, the high concentrations of light-emitting point defects in a colored layer less than $50 \mathrm{~nm}$ thick [30] was enough to obtain a well contrasted fluorescence image. However, as the total number of lightemitting defects decreases in smallest volumes at nanoscale, the need of higher PL signal pushes to use higher laser excitation power, with the risk of not-negligible temporary and/or permanent photo-bleaching of the investigated color-center based samples [19]. Optical microscopy at LNT can directly enhance the collected PL signal, due to the increased efficiency of the laser induced photoemission process and the reduction of the photo-bleaching effects on the active $F_{2}$ and $F_{3}^{+}$color centers. It can open further interesting perspectives for the mapping of peculiar sharp and intense spectral emission features, like the $F_{3}^{+}$zero-phonon lines [20], superimposed to its broad band, as done for other types of color centers in insulating materials [25]. 


\section{Conclusions}

A brief review of the photoemission properties of broadband light-emitting radiation-induced $F_{2}$ and $F_{3}^{+}$electronic point defects in $\mathrm{LiF}$ was presented. These aggregate color centers are stable and laser-active at RT and their peculiar spectral properties were discussed, with emphasis on their behavior at LNT. A few experimental results about optical absorption and photoluminescence spectroscopy and laser fluorescence microscopy were presented.

Optical spectroscopy allows obtaining information about the $F_{2}$ and $F_{3}^{+}$coloration curves in LiF crystals and films and on their relative formation efficiency. The effects of the irradiation temperature on the selective formation of laser-active $F_{2}$ and $F_{3}^{+}$CCs was chosen as an example. The study of the enhanced formation efficiency of aggregate color centers in polycrystalline $\mathrm{LiF}$ films with respect to single crystals is one of the most promising investigation for nanoscience development.

In combination with confocal laser scanning fluorescence microscopy, the effects of photo-bleaching processes on the emission properties of colored LiF samples were also investigated; the color centers mapping in light-emitting microcomponents and nanostructures realised by focused radiation beams and lasers was presented and discussed.

In conclusions, the control of local formation, stabilization and transformation of radiation-induced light-emitting defect centers is crucial for the development of novel optical active photonic devices and imaging radiation sensors based on LiF material in different forms. Some of the advantages and perspectives of low temperature measurements for advanced laser fluorescence microscopy techniques [26], widely used for the spatial mapping of point defects in insulating materials and based on the optical reading of their visible photoluminescence even at nanoscale resolution, were highlighted.

\section{Acknowledgments}

The authors are indebted with many colleagues and friends for useful discussions and suggestions, among them they are grateful to G. Baldacchini, H.J. Kalinowski and E. Nichelatti.

1. J. Nahum and D.A. Wiegand, Phys. Rev. 154, 817 (1967).

2. S.W.S. McKeever, Nucl. Instr. Meth. Phys. Res. B184, 29 (2001).

3. A.R. Lakshmanan, U. Madhusoodanan, A. Natarajan, and B.S. Panigrahy, Phys. Status Solidi A153, 265 (1996).

4. G. Baldacchini, R.M. Montereali, E. Nichelatti, V.S. Kalinov, A.P. Voitovich, A.T. Davidson, and A.G. Kozakiewicz, J. Appl. Phys. 104, 063712 (2008).

5. W. Gellermann, J. Phys. Chem. Solids 52, 1, 249 (1991).

6. T.T. Basiev, S.B. Mirov, and V.V. Osiko, IEEE J. Quantum Electron. 24, 1052 (1988).
7. V.V. Ter-Mikirtychev and T.T. Tsuboi, Prog. Quantum Electron. 20, 3, 219 (1996).

8. R.M. Montereali, Point Defects in Thin Insulating Films of Lithium Fluoride for Optical Microsystems, in: Handbook of Thin Film Materials, H.S. Nalwa (ed.), Academic Press (2002) Vol. 3 Ch.7, p.399.

9. K. Kawamura, M. Horano, T. Kurobori, D. Takamizu, T. Kamiya, and H. Hosono, Appl. Phys. Lett. 84, 311 (2004).

10. G. Baldacchini, F. Bonfigli, A. Faenov, F. Flora, R.M. Montereali, A. Pace, T. Pikuz, and L. Reale, J. Nanoscience Nanotechn. 3, 483 (2003).

11. G. Baldacchini, S. Bollanti, F. Bonfigli, F. Flora, P. Di Lazzaro, A. Lai, T. Marolo, R.M. Montereali, D. Murra, A. Faenov, T. Pikuz, E. Nichelatti, G. Tomassetti, A. Reale, L. Reale, A. Ritucci, T. Limongi, L. Palladino, M. Francucci, S. Martellucci, and G. Petrocelli, Rev. Scient. Instr. 76, 113104-1 (2005) [Also in the Virtual J. Biolog. Phys. Res. 10, No. 11, December 1 (2005)].

12. S. Almaviva, F. Bonfigli, I. Franzini, A. Lai, R.M. Montereali, D. Pelliccia, A. Cedola, and S. Lagomarsino, Appl. Phys. Lett. 89, 54102 (2006).

13. M. Matsubayashi, A. Faenov, T. Pikuz, Y. Fukuda, and Y. Kato, Nucl. Instr. Meth. Phys. Res. A622, 637 (2010).

14. G. Baldacchini, E. De Nicola, R.M. Montereali, A. Scacco, and V. Kalinov, J. Phys. Chem. Solids 61, 21 (2000).

15. R.M. Montereali, S. Bigotta, M. Piccinini, M. Giammatteo, P. Picozzi, and S. Santucci, Nucl. Instr. Meth. Phys. Res. B166-167, 764 (2000).

16. P. Adam, S. Benrezzak, J.L. Bijeon, P. Royer, S. Guy, B. Jacquier, P. Moretti, R.M. Montereali, M. Piccinini, F. Menchini, F. Somma, C. Seassal, and H. Rigneault, Opt. Express 9, 353 (2001).

17. G. Baldacchini, M. Cremona, G. d'Auria, S. Martelli, R.M. Montereali, M. Montecchi, E. Burattini, A. Grilli, and A. Raco, Nucl. Instr. Meth. Phys. Res. B116, 447 (1996).

18. M.A. Vincenti, S. Almaviva, R.M. Montereali, H.J. Kalinowski, and R.N. Nogueira, Appl. Phys. Lett. 89, 241125 (2006).

19. R. Larciprete, L. Gregoratti, M. Danailov, M. Kiskinova, R.M. Montereali, and F. Bonfigli, Appl. Phys. Lett. 80, 3862 (2002).

20. W.B. Fowler, Physics of Color Centers, Academic Press, New York-London (1968).

21. A.P. Voitovich, V.S. Kalinov, S.A. Mikhnov, and S.I. Ovsechuk, Sov. J. Quantum Electron. 17, 780 (1987).

22. G. Baldacchini, F. Bonfigli, F. Menchini, and R.M. Montereali, Nucl. Instr. Meth. Phys. Res. B191, 216 (2002).

23. G. Baldacchini, M. Cremona, G. d'Auria, R.M. Montereali, and V. Kalinov, Phys. Rev. B54, 17508 (1996).

24. S.K. Sekatskii and V.S. Letokhov, Appl. Phys. B63, 525 (1996).

25. Y. Deshko and A.A. Gorokhosky, Fiz. Nizk. Temp. 36, 579 (2010) [Low Temp. Phys. 36, 465 (2010)].

26. E. Rittweger, K.Y. Han, S.E. Irvine, C. Eggeling, and S.W. Hell, Nature Photon. 2, 1 (2009). 
27. H. Gu, L. Qi, and L. Wan, Opt. Commun. 67, 237 (1988).

28. T. Marolo, G. Baldacchini, V.S. Kalinov, and R.M. Montereali, Phys. Status Solidi C2, 367 (2005).

29. G. Baldacchini, G. d'Auria, R.M. Montereali, A. Scacco, J. Phys. Condens. Matter 10, 857 (1998).
30. E. Nichelatti, F. Bonfigli, A. Ya Faenov, F. Flora, T. Marolo, M. Montecchi, R.M. Montereali, T. Pikuz, and G. Baldacchini, J. Non-Crystalline Solids 351, 1774 (2005). 